\title{
GENERALIZATIONS AND SPECIALIZATIONS OF GENERATING FUNCTIONS FOR JACOBI, GEGENBAUER, CHEBYSHEV AND LEGENDRE POLYNOMIALS WITH DEFINITE INTEGRALS
}

\author{
Howard S. COHL AND CONNOR MACKENZIE
}

\begin{abstract}
In this paper we generalize and specialize generating functions for classical orthogonal polynomials, namely Jacobi, Gegenbauer, Chebyshev and Legendre polynomials. We derive a generalization of the generating function for Gegenbauer polynomials through extension a two element sequence of generating functions for Jacobi polynomials. Specializations of generating functions are accomplished through the re-expression of Gauss hypergeometric functions in terms of less general functions. Definite integrals which correspond to the presented orthogonal polynomial series expansions are also given.
\end{abstract}

Mathematics subject classification (2010): 33C45, 05A15, 33C05, 34L10, 30E20.

Keywords and phrases: Othogonal polynomials, Generating functions, Gauss hypergeometric function, Eigenfunction expansions, Definite integrals.

\section{REFERENCES}

[1] M. Abramowitz AND I. A. Stegun, Handbook of mathematical functions with formulas, graphs, and mathematical tables, volume 55 of National Bureau of Standards Applied Mathematics Series, U.S. Government Printing Office, Washington, D. C., 1972.

[2] G. E. Andrews, R. Askey, AND R. RoY, Special functions, volume 71 of Encyclopedia of Mathematics and its Applications, Cambridge University Press, Cambridge, 1999.

[3] I. S. Gradshteyn And I. M. RyZhiK, Table of integrals, series, and products, Elsevier/Academic Press, Amsterdam, seventh edition, 2007.

[4] M. E. H. Ismail, Classical and quantum orthogonal polynomials in one variable, volume 98 of Encyclopedia of Mathematics and its Applications, Cambridge University Press, Cambridge, 2005., With two chapters by Walter Van Assche, With a foreword by Richard A. Askey.

[5] A. JAKImOVSKI, A. Sharma, AND J. SzABADOS, Walsh equiconvergence of complex interpolating polynomials, Springer Monographs in Mathematics, Springer, Dordrecht, 2006.

[6] R. Koekoek, P. A. Lesky, AND R. F. SWARTTOUw, Hypergeometric orthogonal polynomials and their q-analogues, Springer Monographs in Mathematics, Springer-Verlag, Berlin, 2010., With a foreword by Tom H. Koornwinder.

[7] W. Magnus, F. Oberhettinger, And R. P. Soni, Formulas and theorems for the special functions of mathematical physics, Third enlarged edition, Die Grundlehren der mathematischen Wissenschaften, Band 52, Springer-Verlag New York, Inc., New York, 1966.

[8] J. C. MAson AND D. C. HANDSCOMB, Chebyshev polynomials, Chapman \& Hall/CRC, Boca Raton, FL, 2003.

[9] F. W. J. Olver, D. W. Lozier, R. F. Boisvert, AND C. W. Clark, editors. NIST handbook of mathematical functions, Cambridge University Press, Cambridge, 2010.

[10] E. D. RainVille, Special functions, The Macmillan Co., New York, 1960.

[11] B. I. Schneider, J. Segura, A. Gil, X. Guan, And K. Bartschat, A new Fortran 90 program to compute regular and irregular associated Legendre functions, Computer Physics Communications. An International Journal and Program Library for Computational Physics and Physical Chemistry, 181 (12): 2091-2097, 2010. 
[12] H. M. SRIVAstaVa And H. L. Manocha, A treatise on generating functions, Ellis Horwood Series: Mathematics and its Applications, Ellis Horwood Ltd., Chichester, 1984.

[13] G. SzEgő, Orthogonal polynomials, American Mathematical Society Colloquium Publications, Vol. 23. Revised ed. American Mathematical Society, Providence, R.I., 1959.

[14] J. WAN AND W. ZUDILIN, Generating functions of Legendre polynomials: a tribute to Fred Brafman, Journal of Approximation Theory, 164 (4): 488-503, 2012. 